\title{
Initial dip predicts renal protective effects after the administration of sodium-glucose cotransporter 2 inhibitors in patients with type 2 diabetes and chronic kidney disease with normoalbuminuria
}

Kiyohiko Takahashi

Obihiro Kosei Hospital

Akinobu Nakamura ( $\sim$ akinbo@tim.hi-ho.ne.jp )

Hokkaido University https://orcid.org/0000-0002-8192-0006

Sho Furusawa

Obihiro Kosei Hospital

Kei Yokozeki

Obihiro Kosei Hospital

Hajime Sugawara

Obihiro Kosei Hospital

Hideyuki Yanagisawa

Obihiro Kosei Hospital

Kazumasa Akikawa

Obihiro Kosei Hospital

Hideaki Kikuchi

Obihiro Kosei Hospital

Research

Keywords: Chronic kidney disease, Diabetic kidney disease, Normoalbuminuria, Sodium-glucose cotransporter 2 inhibitor

Posted Date: May 28th, 2020

DOl: https://doi.org/10.21203/rs.3.rs-31068/v1

License: (9) (1) This work is licensed under a Creative Commons Attribution 4.0 International License.

Read Full License 
Version of Record: A version of this preprint was published at Journal of Clinical \& Translational Endocrinology on December 1st, 2020. See the published version at https://doi.org/10.1016/j.jcte.2020.100244. 
1 Original Article

2 Initial dip predicts renal protective effects after the administration of sodium-glucose

3 cotransporter 2 inhibitors in patients with type 2 diabetes and chronic kidney disease

$4 \quad$ with normoalbuminuria

5

6 Kiyohiko Takahashi, ${ }^{1}$ Akinobu Nakamura, ${ }^{2}$ Sho Furusawa, ${ }^{1}$ Kei Yokozeki, ${ }^{1}$ Hajime

7 Sugawara, ${ }^{1}$ Hideyuki Yanagisawa, ${ }^{1}$ Kazumasa Akikawa, ${ }^{1}$ and Hideaki Kikuchi ${ }^{1}$

$9 \quad{ }^{1}$ Third Department of Internal Medicine, Obihiro Kosei Hospital, Obihiro, Japan

$10{ }^{2}$ Department of Rheumatology, Endocrinology and Nephrology, Faculty of Medicine

11 and Graduate School of Medicine, Hokkaido University, Sapporo, Japan

13 Corresponding author: Akinobu Nakamura, M.D., Ph.D., Department of Rheumatology,

14 Endocrinology and Nephrology, Faculty of Medicine and Graduate School of Medicine,

15 Hokkaido University, Sapporo, Japan

16 N-15, W-7, Kita-ku, Sapporo 060-8638, Japan

17 Tel: +81-11-706-5915

18 Fax: +81-11-706-7710 
19 E-mail: akinbo@tim.hi-ho.ne.jp

20 


\section{Abstract}

22 Background: We investigated the renoprotective effects of sodium-glucose

23 cotransporter 2 inhibitors (SGLT2is) on renal function in patients with type 2 diabetes

24 and chronic kidney disease (CKD) with normoalbuminuria.

25 Methods: A retrospective review of clinical records of Japanese participants with type 2 diabetes and CKD (estimated glomerular filtration rate $[\mathrm{eGFR}]<60 \mathrm{~mL} / \mathrm{min} / 1.73 \mathrm{~m}^{2}$ ) with normoalbuminuria (urine albumin to creatinine ratio $<30 \mathrm{mg} / \mathrm{g} \mathrm{Cr}$ and/or urinary protein to creatinine ratio $<150 \mathrm{mg} / \mathrm{g} \mathrm{Cr}$ ) was conducted. Participants were categorized into two groups depending on whether they had started using SGLT2is. The main study outcome was a comparison of the change in renal function evaluated by eGFR after 1 year $(\triangle \mathrm{eGFR}+1 \mathrm{y})$ between the two groups. Then, we identified predictors that were associated with the outcome.

Results: Among the 48 participants, 21 were treated with SGLT2is (SGLT2 group) and 25 were treated with other antidiabetic medications (control group). Although eGFR was significantly decreased at 1 year in the control group, the decline in eGFR was not observed in the SGLT2 group. The change in eGFR was significantly greater in the SGLT2 group than in the control group $(\triangle \mathrm{eGFR}+1$ year, $-4.0[-7.7$ to -0.3$]$ $\mathrm{mL} / \mathrm{min} / 1.73 \mathrm{~m}^{2}$ in the control group, $0.9[-3.9$ to 5.7$] \mathrm{mL} / \mathrm{min} / 1.73 \mathrm{~m}^{2}$ in the SGLT2 
39 group; $P=0.0231$ ). Additionally, multiple linear regression analysis showed that an

40 initial dip was an independent factor associated with the worsening of renal function in

41 the SGLT2 group.

42 Conclusions: Although more favorable effects of SGLT2is on renal function were

43 observed in patients with type 2 diabetes and CKD with normoalbuminuria, the higher

44 initial dip was a possible marker of worsening renal function after the initiation of

45 SGLT2is.

46

47

48 Keywords: Chronic kidney disease, Diabetic kidney disease, Normoalbuminuria,

49 Sodium-glucose cotransporter 2 inhibitor

50 


\section{Background}

The incidence and prevalence of diabetes are increasing worldwide, and the ultimate goal of treating diabetes is to prevent or delay the progression of microvascular and macrovascular complications. Dialysis resulting from diabetes is extremely prevalent in Japan [1]. In 2017, the number of patients on chronic dialysis reached 334,505 [1]. Traditionally, it has been thought that typical diabetic nephropathy progresses from normal albuminuria to micro/macroalbuminuria and gradually to decreases in renal function. However, recently, it has been shown that there are atypical diabetic nephropathy cases that already exhibit reduced renal function without micro/macroalbuminuria. Therefore, diabetic kidney disease was proposed as a condition that includes typical and atypical diabetic nephropathy [2].

Sodium-glucose cotransporter 2 inhibitors (SGLT2is) improve glucose tolerance by suppressing renal glucose reabsorption without direct pharmacological action on pancreatic beta cells. Four large prospective clinical trials have shown that SGLT2is decrease the composition renal endpoints such as decline in estimated glomerular filtration rate (eGFR), doubling of the serum creatinine level, new end-stage kidney disease, death from renal or cardiovascular causes, or progression to macroalbuminuria [3-6]. In addition, it has been reported that, in patients with type 2 diabetes and chronic 
69 kidney disease (CKD), SGLT2is prevent the decline in eGFR [7-10]. However, studies

70 on the effects of SGLT2is on renal function in patients with type 2 diabetes and CKD

71 with normoalbuminuria remain limited $[8,9]$.

72 In the present study, we investigated the renoprotective effects of SGLT2is on renal

73 function in patients with type 2 diabetes and CKD with normoalbuminuria and

74 identified predictors that were associated with the outcome. 


\section{Methods}

\section{Study design and participants}

The major inclusion criteria were type 2 diabetes and CKD (eGFR $<60$

$\mathrm{mL} / \mathrm{min} / 1.73 \mathrm{~m}^{2}$ ) with normoalbuminuria (urine albumin to creatinine ratio [UACR] <

$30 \mathrm{mg} / \mathrm{g} \mathrm{Cr}$ and/or urinary protein to creatinine ratio [UPCR] $<150 \mathrm{mg} / \mathrm{g} \mathrm{Cr}$ ) at

SGLT2is initiation. As a control, patients who were not administered SGLT2is were also

included. The exclusion criteria were as follows: type 1 diabetes, use of glucagon-like

peptide- 1 receptor agonists for type 2 diabetes, endocrine disease, use of steroids or

immunosuppressants for autoimmune disease, dialysis, transplantation, liver cirrhosis,

and malignancy. Finally, 46 patients were eligible for evaluation. The study was

conducted with the approval of the Institutional Review Board of Obihiro Kosei

Hospital (2020-017), and registered with the University Hospital Medical Information

Network (UMIN; number UMIN000040424).

\section{Study definitions and outcomes}

94 We conducted a retrospective review of the clinical records of all consecutive 
depending on whether they used SGLT2is: in the SGLT2 group, patients started receiving SGLT2is in addition to their other antidiabetic medication; and in the control group, patients received conventional antidiabetic medications alone. Outcome data were collected from the patients' medical records. Baseline data for age, sex, duration of diabetes, and medications for diabetes and hypertension were also collected. Common measurements, such as body weight, body mass index (BMI), systolic and diastolic blood pressure (SBP and DBP, respectively), plasma glucose (PG) level, glycated hemoglobin (HbA1c) level, kidney and liver function test results, high-density lipoprotein cholesterol (HDL) level, low-density lipoprotein (LDL) level, triglyceride (TG) level, UACR, and/or UPCR, at each clinic visit were collected. The eGFR 1-2 months after SGLT2is initiation was also used as an eGFR initial data point. We defined the change in eGFR as the initial dip, as previously reported $[7,10,11]$. These parameters were measured using commercially available assay kits. The primary objective of this study was to assess the clinical effectiveness of SGLT2is on renal function by analyzing the change in eGFR at one year $(\Delta \mathrm{GFR}+1 \mathrm{y})$ after initiating SGLT2is, compared with the change after conventional antidiabetic medication. The secondary objective was to investigate the variables associated with $\Delta \mathrm{GFR}+1 \mathrm{y}$, to identify suitable patients with type 2 diabetes for the renoprotective 
115 effect of SGLT2is. The initial dip and $\Delta \mathrm{GFR}+1$ y were calculated as follows: initial

$116 \mathrm{dip}=\mathrm{eGFR}$ at 1 or 2 months after starting SGLT2is - eGFR at the start of SGLT2is;

$117 \Delta \mathrm{GFR}+1 \mathrm{y}=\mathrm{eGFR}$ at 1 year $-\mathrm{eGFR}$ at baseline

\section{Statistical analysis}

119 Chi-square test, Mann-Whitney $U$-test, unpaired $t$-test, or paired $t$-test was used to

120 compare between the two groups as appropriate. Results are shown as mean \pm standard

121 deviation or median. We used a two-way analysis of variance (ANOVA) followed by

122 post hoc Bonferroni test for repeated measurements. Calculations for correlation

123 coefficients and simple linear regression analyses were performed to test for

124 associations between $\triangle \mathrm{GFR}+1 \mathrm{y}$ and baseline parameters in the SGLT2 group.

125 Additionally, we performed stepwise multivariate regression analysis to examine which

126 factors independently determined $\Delta \mathrm{GFR}+1 \mathrm{y}$ in the SGLT2 group. $P$-values $<0.05$ were

127 considered statistically significant. Statistical analyses were performed using JMP 14

128 (SAS Inc., Cary, NC, USA) and Microsoft Excel Statistics 2012 for Windows (SSRI

129 Co. Ltd, Tokyo, Japan). 
Table 1 shows the underlying diseases present in study patients. Of the 46 patients,

133 the SGLT2 group comprised 21 patients, and the control group comprised 25 patients.

134 There were no differences in most of the baseline parameters between the two groups,

135 except for duration of diabetes, PG, HbA1c, alanine aminotransferase (ALT), or TG.

136 The number of patients with CKD staging among both groups was as follows: SGLT2

137 group G3a: 15, G3b: 5, and G4: 1; control group G3a: 18, G3b: 5, and G4: 2. As shown

138 in Fig. 1, the eGFR in the control group was significantly decreased after 1 year.

139 However, there was no decrease in eGFR in the SGLT2 group. As shown in Fig. 2, the

140 magnitude of the effect for eGFR was significantly greater in the SGLT2 group than in

141 the control group $\left(\Delta \mathrm{eGFR}+1\right.$ year: $-4.0(-7.7$ to -0.3$) \mathrm{mL} / \mathrm{min} / 1.73 \mathrm{~m}^{2}$ in the control

142 group, $0.9(-3.9$ to 5.7$) \mathrm{mL} / \mathrm{min} / 1.73 \mathrm{~m}^{2}$ in the SGLT2 group; $\left.P=0.0231\right)$. Next, to

143 reveal the factors associated with the changes in eGFR at 1 year after starting SGLT2is,

144 we examined the correlations between $\triangle \mathrm{eGFR}+1$ year and the clinical parameters in

145 the SGLT2 group. As shown in Table 2, only the initial dip showed a significant

146 positive correlation $(P=0.0159)$ with $\triangle \mathrm{eGFR}+1$ year. Moreover, as shown in Table 3 ,

147 multiple linear regression analysis identified higher initial dip as an independent factor associated with worsening of renal function in the SGLT2 group, after adjusting for age, 
149 BMI, HbA1c level, and eGFR $(P=0.0166)$.

150

151

152

153

154

155

156

157

158

159

160

161

162

163

164

165

166 


\section{Discussion}

In the present study, we showed that additional treatment with SGLT2is prevents the

169 decline in eGFR in the SGLT2 group compared with that in the control group. Although we had previously shown that more favorable effects of SGLT2is on renal function in

171 patients with type 2 diabetes and CKD with normoalbuminuria compared with patients

172 with macroalbuminuria [9], this study did not include a control group. Therefore, this

173 study was able to confirm the renoprotective effects on SGLT2is for patients with type 2

diabetes and CKD with normoalbuminuria compared with non-SGLT2is user.

In addition, using multiple linear regression analysis we showed that the initial dip in the SGLT2 group was useful in predicting the renoprotective effect of SGLT2is.

Previously, Miyoshi et al. showed that the decline in eGFR before starting SGLT2is in patients with type 2 diabetes and CKD stages 3-4 was an independent factor associated with renal function outcomes [7]. However, there have been no studies on the predictive factors associated with the worsening of renal function in patients with type 2 diabetes and CKD with normoalbuminuria. This is the first report to identify independent factors associated with the worsening of renal function in patients with type 2 diabetes and

CKD with normoalbuminuria. 
185 is decreasing intraglomerular pressure through tubuloglomerular feedback restoration

186 [12-15]. It has also been reported that empagliflozin reduces the intraglomerular

187 pressure to 6-8 $\mathrm{mmHg}$ in patients with type 1 diabetes [16]. In fact, a decrease in eGFR

188 is typically observed within 1 month after initiating SGLT2is therapy [3]. This

189 phenomenon is called the initial dip or initial drop [17]. As other mechanisms, SGLT2is

190 have the renoprotective effect through not only their anti-inflammatory and

191 anti-oxidative stress effects [18, 19], but also increase of hematocrit and

$192 \beta$-hydroxybutyrate [15].

193 Pathological findings revealed that tubulointerstitial and vascular lesions were more

194 advanced in patients with type 2 diabetes and CKD with normoalbuminuria than in

195 those with micro/macroalbuminuria [20]. In contrast, glomerular lesions were more

196 advanced in patients with type 2 diabetes and CKD with micro/macroalbuminuria than

197 in those with normoalbuminuria [20]. Previously, it has been shown that the

198 pathological findings in patients with type 2 diabetes and CKD with normoalbuminuria

199 are similar to those in patients with nephrosclerosis [21]. In nephrosclerosis, it is

200 thought that the glomeruli undergo progressive ischemic changes [22], in which

201 intraglomerular pressure can be normal or decreased [23]. Results from the EMPA-REG

202 outcome study demonstrated that the eGFR increases after withdrawing SGLT2i [3]. 
203 These data support the idea that the initial dip in GFR after SGLT2is administration is a

204 hemodynamic effect [3]. It seems likely that some patients with type 2 diabetes and

205 CKD with normoalbuminuria who show a high initial dip after SGLT2is initiation have

206 reduced renoprotective effects due to an excessive drop in intraglomerular pressure

207 caused by changes in blood volume or renal perfusion. Future studies will determine

208 whether this result can also be applied to patients with type 2 diabetes and CKD with

$209 \mathrm{micro} / \mathrm{macroalbuminuria}$ with glomerular lesions.

210 There are some limitations to the present study. First, this study was retrospective in

211 nature. Second, the sample size was small, which might have limited its statistical

212 power. Third, baseline parameters such as the duration of diabetes, PG levels, and

213 HbA1c levels were unbalanced between the groups, which may have made

214 interpretation difficult. Fourth, this study had a short follow-up period and was

215 conducted in a single center. Larger and longer prospective studies will be required to

216 verify our results in the future.

217 In conclusion, our study suggests that renoprotective effects of SGLT2is on renal

218 function were observed in patients with type 2 diabetes with CKD and

219 normoalbuminuria. Additionally, patients who show a high initial dip after SGLT2is

initiation should be carefully monitored for decline in renal function. 


\section{Abbreviations}

222 ACEI, angiotensin-converting enzyme inhibitor; ALT, alanine aminotransferase; ARB,

223 angiotensin II receptor blocker; AST, aspartate aminotransferase; BMI, body mass

224 index; CKD, chronic kidney disease; DBP, diastolic blood pressure; eGFR, estimated

225 glomerular filtration rate; HbA1c, glycated hemoglobin; HDL, high-density lipoprotein

226 cholesterol; LDL, low-density lipoprotein; PG, plasma glucose; SBP, systolic blood

227 pressure; SGLT2is, sodium-glucose cotransporter 2 inhibitors; TG, triglyceride; UACR,

228 urinary albumin-to-creatinine ratio; UPCR, urinary protein to creatinine ratio.

\section{$230 \quad$ Declarations}

231 Ethics approval and consent to participate: This study was conducted with approval

232 from the Institutional Review Board of the Obihiro Kosei Hospital (2020-017), and

233 registered with the University Hospital Medical Information Network (UMIN; number

234 UMIN000040424).

235 Consent for publication: Not applicable.

236 Availability of data and materials: The datasets used and/or analyzed during the

237 current study are available from the corresponding author on reasonable request.

238 Competing interests: AN has obtained research support from Mitsubishi Tanabe 
239 Pharma, Daiichi Sankyo, MSD, Novo Nordisk Pharma, Novartis Pharma, AstraZeneca,

240 LifeScan Japan, Nippon Boehringer Ingelheim, and Taisho Pharmaceutical.

241 Funding: This study was not supported by any institution.

242 Authors' contributions: KT and AN contributed to writing the manuscript. KT and SF

243 contributed to data analysis. KT, AN, and SF contributed to the discussion, and

244 reviewed and edited the manuscript. KT is the guarantor of this work and, as such, had

245 full access to all data in the study and takes responsibility for the integrity of the data

246 and accuracy of the data analysis. All authors have read and approved the final

247 manuscript.

248 Acknowledgments: We thank the participants at Obihiro Kosei Hospital.

249

250 


\section{References}

252 1. Nitta K, Masakane I, Hanafusa N, et al. Annual dialysis data report 2017, JSDT renal data registry. Ren Replace Ther. 2019; 5: 1-44.

2. Tuttle KR, Bakris GL, Bilous RW, et al. Diabetic kidney disease: a report from an ADA Consensus Conference. Diabetes Care. 2014; 37: 2864-83.

3. Wanner C, Inzucchi SE, Lachin JM, et al. Empagliflozin and progression of kidney disease in type 2 diabetes. N Engl J Med. 2016; 375: 323-34.

4. Neal B, Perkovic V, Mahaffey KW, et al. Canagliflozin and cardiovascular and renal events in type 2 diabetes. N Engl J Med. 2017; 377: 644-57.

5. Wiviott SD, Raz I, Bonaca MP, et al. Dapagliflozin and cardiovascular outcomes in type 2 diabetes. N Engl J Med. 2019; 380: 347-57.

6. Perkovic V, Jardine MJ, Neal B, et al. Canagliflozin and renal outcomes in type 2 diabetes and nephropathy. N Engl J Med. 2019; 380: 2295-306.

7. Miyoshi H, Kameda H, Yamashita K, et al. Protective effect of sodium-glucose cotransporter 2 inhibitors in patients with rapid renal function decline, stage G3 or G4 chronic kidney disease and type 2 diabetes. J Diabetes Investig. 2019; 10: 1510-7.

8. Sugiyama S, Jinnouchi H, Yoshida A, et al. Renoprotective effects of additional SGLT2 inhibitor therapy in patients with type 2 diabetes mellitus and chronic kidney disease stages 3b-4: a real world report from a Japanese specialized diabetes care center. J Clin Med Res. 2019; 11: 267-74.

9. Nakamura A, Miyoshi $\mathrm{H}$, Kameda $\mathrm{H}$, et al. Impact of sodium-glucose cotransporter 2 inhibitors on renal function in participants with type 2 diabetes and chronic kidney disease with normoalbuminuria. Diabetol Metab Syndr. 2020; 12: 4.

10. Yale JF, Bakris G, Cariou B, et al. Efficacy and safety of canagliflozin in subjects with type 2 diabetes and chronic kidney disease. Diabetes Obes Metab. 2013; 15: 463-73.

11. Cherney DZ, Perkins BA, Soleymanlou N, et al. Renal hemodynamic effect of sodium-glucose cotransporter 2 inhibition in patients with type 1 diabetes mellitus. Circulation. 2014; 129: 587-97.

12. Musso G, Gambino R, Cassader M, et al. A novel approach to control hyperglycemia in type 2 diabetes: sodium glucose co-transport (SGLT) inhibitors: systematic review and meta-analysis of randomized trials. Ann Med. 2012; 44: 375-93. 
13. Cherney DZ, Perkins BA, Soleymanlou N, et al. The effect of empagliflozin on arterial stiffness and heart rate variability in subjects with uncomplicated type 1 diabetes mellitus. Cardiovasc Diabetol. 2014; 13: 28.

14. Stanton RC. Sodium glucose transport 2 (SGLT2) inhibition decreases glomerular hyperfiltration: is there a role for SGLT2 inhibitors in diabetic kidney disease? Circulation. 2014; 129: 542-4.

15. Mima A. Renal protection by sodium-glucose cotransporter 2 inhibitors and its underlying mechanisms in diabetic kidney disease. J Diabetes Complications. 2018; 32: 720-5.

16. Skrtic M, Yang GK, Perkins BA, et al. Characterisation of glomerular haemodynamic responses to SGLT2 inhibition in patients with type 1 diabetes and renal hyperfiltration. Diabetologia. 2014; 57: 2599-602.

17. Vallon V, Thomson SC. Targeting renal glucose reabsorption to treat hyperglycaemia: the pleiotropic effects of SGLT2 inhibition. Diabetologia. 2017; 60: 215-25.

18. Dekkers CCJ, Petrykiv S, Laverman GD, et al. Effects of the SGLT-2 inhibitor dapagliflozin on glomerular and tubular injury markers. Diabetes Obes Metab. 2018; 20: 1988-93.

19. Ishibashi Y, Matsui T, Yamagishi S. Tofogliflozin, A highly selective inhibitor of SGLT2 blocks proinflammatory and proapoptotic effects of glucose overload on proximal tubular cells partly by suppressing oxidative stress generation. Horm Metab Res. 2016; 48: 191-5.

20. Shimizu M, Furuichi K, Toyama T, et al. Long-term outcomes of Japanese type 2 diabetic patients with biopsy-proven diabetic nephropathy. Diabetes Care. 2013; 36: 3655-62.

21. Hill GS. Hypertensive nephrosclerosis. Curr Opin Nephrol Hypertens. 2008; 17: 266-70.

22. Preston RA, Epstein M. Ischemic renal disease: an emerging cause of chronic renal failure and end-stage renal disease. J Hypertens. 1997; 15: 1365-77.

23. Abe M, Soma M. Multifunctional L/N- and L/T-type calcium channel blockers for kidney protection. Hypertens Res. 2015; 38: 804-6. 
$323 \quad$ Fig. 1

324 Changes in eGFR in patients with or without SGLT2is administration. Data are 325 presented as mean \pm standard deviation $(\mathrm{SD}) .{ }^{*} P<0.01$ : two-way analysis of variance 326 followed by post hoc Bonferroni test, 0 year vs. 1 year in the control group. eGFR, 327 estimated glomerular filtration rate; SGLT2is, sodium-glucose cotransporter 2 328 inhibitors.

329 Fig. 2

330 Comparison of changes in eGFR in patients with or without SGLT2is administration.

331 The Wilcoxon rank-sum test was used for statistical analysis. $* P<0.05$. eGFR, 332 estimated glomerular filtration rate; SGLT2is, sodium-glucose cotransporter 2 333 inhibitors. 
Table 1. Baseline characteristics of the 46 participants

\begin{tabular}{|c|c|c|c|}
\hline & Control $(n=25)$ & SGLT2 $(n=21)$ & $P$ value \\
\hline Age (years) & $67.9 \pm 9.1$ & $67.4 \pm 7.9$ & 0.8333 \\
\hline Man/Woman (n) & $12 / 13$ & $11 / 10$ & 0.7672 \\
\hline Duration of diabetes (years) & $7.0(2.0$ to 13.5$)$ & $12.5(7.3$ to 24.0$)$ & 0.0429 \\
\hline Weight (kg) & $65.3 \pm 14.5$ & $69.9 \pm 12.1$ & 0.2810 \\
\hline $\operatorname{BMI}\left(\mathrm{kg} / \mathrm{m}^{2}\right)$ & $25.5 \pm 4.2$ & $27.9 \pm 4.7$ & 0.0967 \\
\hline PG (mg/dl) & $146.8 \pm 47.4$ & $206.1 \pm 79.6$ & 0.0032 \\
\hline $\operatorname{HbA1c}(\%)$ & $7.1 \pm 1.0$ & $8.0 \pm 1.1$ & 0.0058 \\
\hline $\mathrm{eGFR}\left(\mathrm{mL} / \mathrm{min} / 1.73 \mathrm{~m}^{2}\right)$ & $49.3 \pm 10.9$ & $49.7 \pm 9.1$ & 0.9014 \\
\hline AST (U/L) & $22.0(18.5$ to 28.0$)$ & $26.0(20.0$ to 42.0$)$ & 0.0898 \\
\hline ALT (U/L) & $23.0(13.0$ to 30.0$)$ & $29.0(20.0$ to 38.0$)$ & 0.0310 \\
\hline $\mathrm{HDL}(\mathrm{mg} / \mathrm{dL})$ & $45.1 \pm 13.0$ & $49.7 \pm 15.1$ & 0.2875 \\
\hline LDL (mg/dL) & $109.7 \pm 33.4$ & $106.1 \pm 26.2$ & 0.6995 \\
\hline $\mathrm{TG}(\mathrm{mg} / \mathrm{dL})$ & 170.5 (126.3 to 222.8$)$ & $121.0(92.0$ to 163.5$)$ & 0.0224 \\
\hline Stage of diabetic nephropathy & III 23/ IV 2 & III 20/ IV 1 & 0.6577 \\
\hline Chronic kidney disease & IIIa 18/IIIb 5/ IV 2 & IIIa 15/ IIIb 5/ IV 1 & 0.8780 \\
\hline UACR (mg/g Cr) & $13.0(7.3$ to 16.9$)$ & $12.6(8.0$ to 16.5$)$ & 0.8838 \\
\hline $\mathrm{UPCR}(\mathrm{mg} / \mathrm{g} \mathrm{Cr})(\mathrm{n}=1$ and 4$)$ & 50.8 & $115.21 \pm 21.7$ & - \\
\hline SBP (mmHg) & $126.3 \pm 22.0$ & $127.2 \pm 10.8$ & 0.9058 \\
\hline DBP (mmHg) & $73.5 \pm 14.5$ & $70.91 \pm 9.1$ & 0.6085 \\
\hline Treatment with ARB or ACEI (\%) & 56.0 & 47.6 & 0.5708 \\
\hline Diuretics (\%) & 24.0 & 14.3 & 0.4081 \\
\hline \multicolumn{4}{|l|}{ SGLT2i (\%) } \\
\hline Empagliflozin & 0 & 28.6 & \\
\hline Ipragliflozin & 0 & 14.3 & \\
\hline Luseogliflozin & 0 & 0 & \\
\hline Dapagliflozin & 0 & 19.1 & \\
\hline Tofogliflozin & 0 & 0 & \\
\hline Canagliflozin & 0 & 38.1 & \\
\hline \multicolumn{4}{|l|}{ Antidiabetic drugs (\%) } \\
\hline Sulfonylureas (\%) & 16.0 & 4.8 & 0.2226 \\
\hline Metformin $(\%)$ & 32.0 & 33.3 & 0.9235 \\
\hline Thiazolidinedione (\%) & 0 & 0 & - \\
\hline Alpha-glucosidase inhibitor (\%) & 4.0 & 23.8 & 0.0469 \\
\hline Glinide (\%) & 8.0 & 19.1 & 0.2678 \\
\hline
\end{tabular}




\begin{tabular}{lllc} 
Dipeptidyl peptidase-4 inhibitor (\%) & 84.0 & 71.4 & 0.3032 \\
Insulin (\%) & 24.0 & 28.6 & 0.7251 \\
Glucagon like peptide-1 receptor agonist (\%) & 0 & 0 & - \\
\hline
\end{tabular}

345 Values are shown as means \pm standard deviation, median, $\mathrm{n}$, or $\%$.

346 SGLT2, sodium-glucose cotransporter 2; BMI, body mass index; PG, plasma glucose;

347 HbA1c, glycated hemoglobin; eGFR, estimated glomerular filtration rate; AST,

348 aspartate aminotransferase; ALT, alanine aminotransferase; HDL, high-density

349 lipoprotein cholesterol; LDL, low-density lipoprotein; TG, triglyceride; UACR, urine

350 albumin-to-creatinine ratio; UPCR, urinary protein to creatinine ratio; SBP, systolic

351 blood pressure; DBP, diastolic blood pressure; ARB, angiotensin II receptor blocker;

352 ACEI, angiotensin-converting enzyme inhibitor; SGLT2i, sodium-glucose cotransporter

3532 inhibitor.

354

355

356

357

358

359

360

361

362

363

364

365

366

367

368 
SGLT2 group

\begin{tabular}{llr}
\hline & Correlation coefficient & $P$ value \\
\hline Duration of diabetes (years) & 0.1701 & 0.4735 \\
Age (years) & -0.0839 & 0.7178 \\
Weight $(\mathrm{kg})$ & -0.2120 & 0.3983 \\
BMI $\left(\mathrm{kg} / \mathrm{m}^{2}\right)$ & -0.2047 & 0.4153 \\
PG $(\mathrm{mg} / \mathrm{dl})$ & 0.0562 & 0.8089 \\
HbA1c $(\%)$ & 0.2048 & 0.3731 \\
eGFR $\left(\mathrm{mL} / \mathrm{min} / 1.73 \mathrm{~m}^{2}\right)$ & -0.1697 & 0.4621 \\
initial dip $\left(\mathrm{mL} / \mathrm{min} / 1.73 \mathrm{~m}^{2}\right)$ & 0.5448 & 0.0159 \\
AST $(\mathrm{U} / \mathrm{L})$ & 0.1409 & 0.5649 \\
ALT $(\mathrm{U} / \mathrm{L})$ & 0.0723 & 0.7688 \\
HDL $(\mathrm{mg} / \mathrm{dL})$ & -0.1405 & 0.5436 \\
LDL $(\mathrm{mg} / \mathrm{dL})$ & 0.2012 & 0.4089 \\
TG $(\mathrm{mg} / \mathrm{dL})$ & 0.1953 & 0.3963 \\
$\mathrm{UACR}(\mathrm{mg} / \mathrm{g} \mathrm{Cr})$ & -0.3991 & 0.0813 \\
SBP $(\mathrm{mmHg})$ & 0.5605 & 0.0729 \\
DBP $(\mathrm{mmHg})$ & 0.4721 & 0.1426 \\
\hline
\end{tabular}

$\Delta \mathrm{eGFR}+1 \mathrm{y}$, the change in estimated glomerular filtration rate at one year after starting

sodium-glucose cotransporter 2 inhibitor; SGLT2, sodium-glucose cotransporter 2;

BMI, body mass index; PG, plasma glucose; HbA1c, glycated hemoglobin; eGFR,

estimated glomerular filtration rate; AST, aspartate aminotransferase; ALT, alanine aminotransferase; HDL, high-density lipoprotein cholesterol; LDL, low-density 
379 Table 3. Multiple regression analysis for $\triangle \mathrm{eGFR}+1 \mathrm{y}$ and baseline parameters in the $380 \quad$ SGLT2 group

\begin{tabular}{llr}
\hline & Standardized partial regression coefficient & $P$ value \\
\hline Initial $\operatorname{dip}\left(\mathrm{mL} / \mathrm{min} / 1.73 \mathrm{~m}^{2}\right)$ & 0.7073 & 0.0166 \\
\hline
\end{tabular}

$381 R^{2}=0.2793$. A multiple regression with stepwise selection was performed considering

382 age, BMI, HbA1c, eGFR, and initial dip. $\triangle \mathrm{eGFR}+1 \mathrm{y}$, the change in estimated

383 glomerular filtration rate one year after starting sodium-glucose cotransporter 2

384 inhibitor; SGLT2, sodium-glucose cotransporter 2; BMI, body mass index; HbA1c,

385 glycated hemoglobin; eGFR, estimated glomerular filtration rate.

386

387 


\section{Figures}

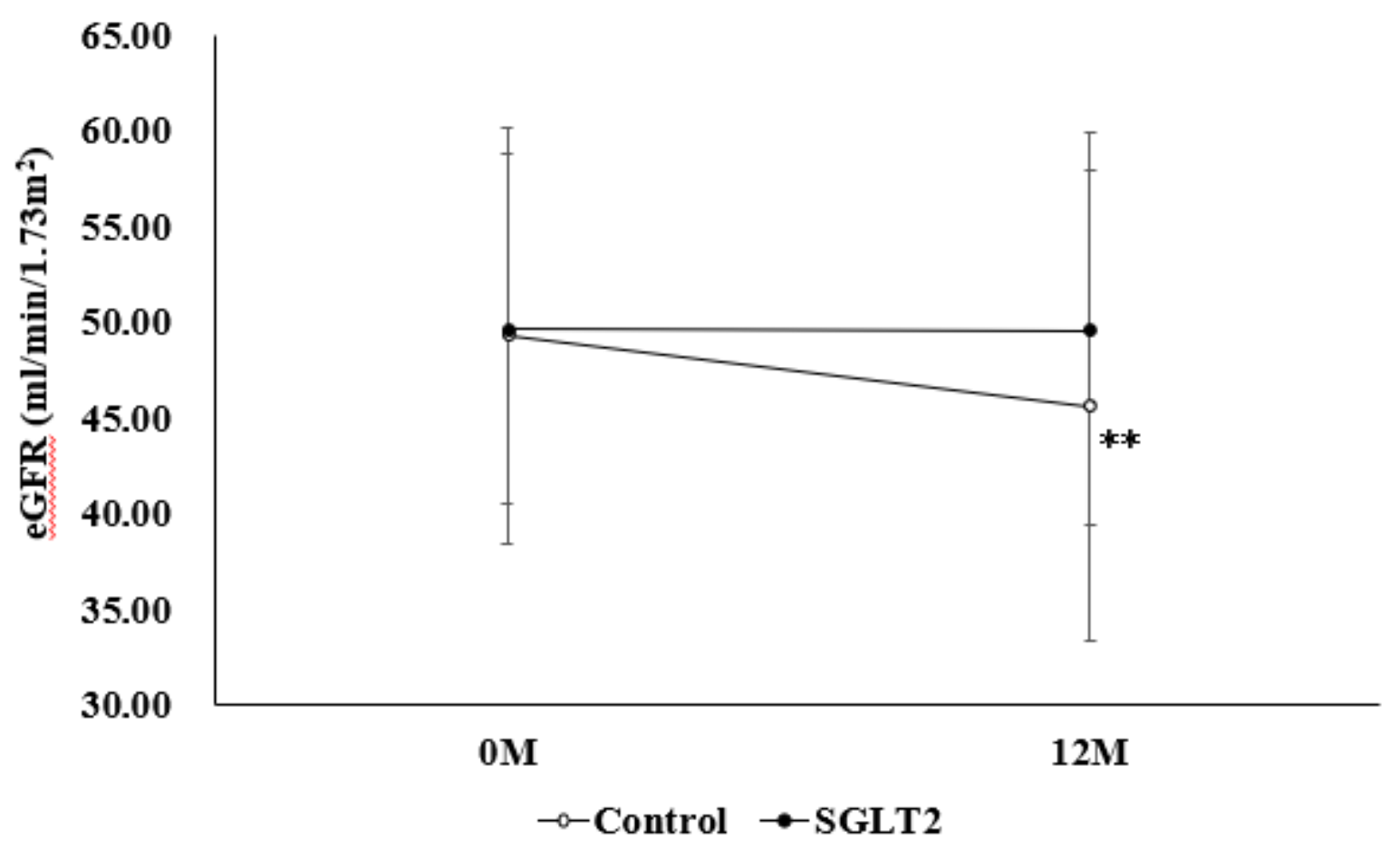

Figure 1

Changes in eGFR in patients with or without SGLT2is administration. Data are presented as mean \pm standard deviation (SD). ${ }^{*} \mathrm{P}<0.01$ : two-way analysis of variance followed by post hoc Bonferroni test, 0 year vs. 1 year in the control group. eGFR, estimated glomerular filtration rate; SGLT2is, sodium-glucose cotransporter 2 inhibitors. 


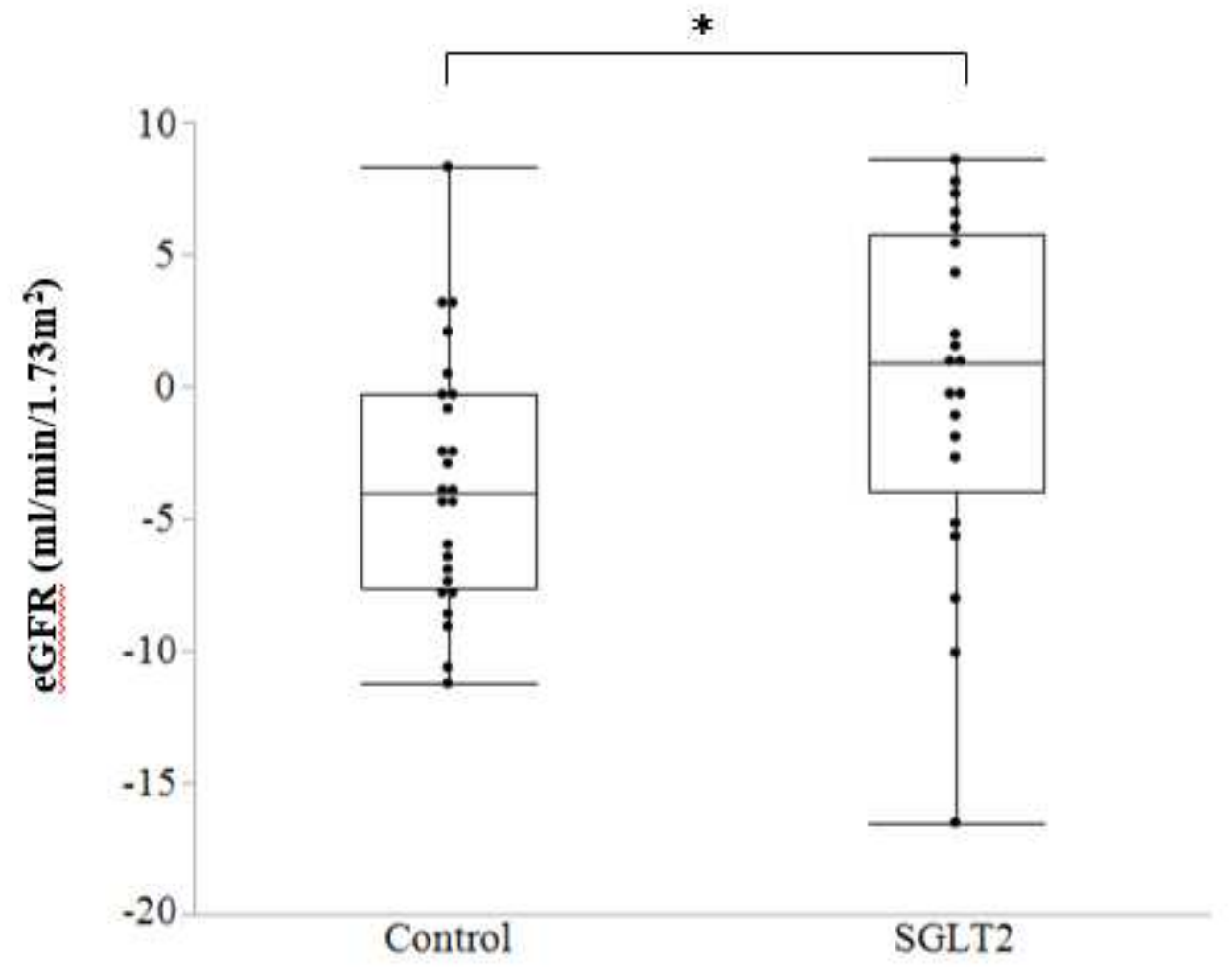

Figure 2

Comparison of changes in eGFR in patients with or without SGLT2is administration. The Wilcoxon ranksum test was used for statistical analysis. ${ }^{*} \mathrm{P}<0.05$. eGFR, estimated glomerular filtration rate; SGLT2is, sodium-glucose cotransporter 2 inhibitors. 